\title{
Review Only
}

\section{Przewalskin A: A Novel $\mathbf{C}_{23}$}

Terpenoid with a 6/6/7 Carbon

Ring Skeleton from Salvia

przewalskii Maxim

Gang Xu, Ai-Jun Hou, Rui-Rui Wang, Guang-Yu Liang, Yong-Tang Zheng, Ze-Yuan

Liu, Xiao-Li Li, Yu Zhao, Sheng-Xiong Huang, Li-Yan Peng, and Qin-Shi Zhao

\section{Supporting Information}

\section{List of Supporting Information}

- Spectroscopic and physical data of $\mathbf{1}$

- Spectroscopic and physical data of 2

- PDC oxidation procedure of $\mathbf{1}$

- 1D and 2D NMR spectra of 1 in acetone- $d_{6}$

- ${ }^{1} \mathrm{H}$ NMR and ${ }^{1} \mathrm{H}-{ }^{1} \mathrm{H}$ ROESY spectra of $\mathbf{1}$ in $\mathrm{C}_{5} \mathrm{D}_{5} \mathrm{~N}$

- 1D and 2D NMR spectra of 2 in $\mathrm{CDCl}_{3}$

- 1D and 2D NMR spectra of 1 in $\mathrm{CDCl}_{3}$ 
Przewalskin A (1): yellow powders; $[\alpha]_{\mathrm{D}}^{20.2}=+100.5^{\circ}\left(c 0.68, \mathrm{CHCl}_{3}\right) ; \mathrm{UV}(\mathrm{MeOH})$ $\lambda_{\max }(\log \varepsilon): 269(1.51), 331(1.20) \mathrm{nm}$; IR (KBr) $v_{\max }: 3443,2928,1632,1590,1526$ 1418, 1637, 1341, 1083, $1015 \mathrm{~cm}^{-1}$; NMR can be found in Table 1; EIMS $\mathrm{m} / z$ (rel. int.): 402 ([M] $\left.]^{+}, 19\right), 384$ (5), 356 (92), 355 (100), 339 (20), 338 (19), 313 (34), 299 (17), 285 (30), 257 (18); HR-ESIMS found 425.1949, calcd for $\mathrm{C}_{23} \mathrm{H}_{30} \mathrm{O}_{6} \mathrm{Na} 425.1940$.

Przewalskin B (2): yellow needles (from MeOH). M.p. $202-204{ }^{\circ} \mathrm{C} ;[\alpha]_{\mathrm{D}}^{21.8}=+164.1^{\circ}$ (c 0.77, $\left.\mathrm{CHCl}_{3}\right) ; \mathrm{UV}(\mathrm{MeOH}) \lambda_{\max }(\log \varepsilon): 239$ (3.82), 276 (3.92), 324 (3.56) nm; IR (KBr) $v_{\max }: 3435,2924,1755,1653,1585,1466,1415,1372,1342,1231,1158 \mathrm{~cm}^{-1}$; NMR can be found in Table 2; EIMS m/z (rel. int.): 398 ([M] $\left.]^{+}, 18\right), 371$ (17), 370 (74), 355 (9), 342 (56), 341 (70), 327 (23), 299 (67), 115 (29), 83 (100); HR-ESIMS found 421.1619, calcd for $\mathrm{C}_{23} \mathrm{H}_{26} \mathrm{O}_{6} \mathrm{Na} 425.1627$.

\section{PDC oxidation procedure of 1:}

PDC (25 mg) was added to the $\mathrm{CH}_{2} \mathrm{Cl}_{2}$ solution $(2.0 \mathrm{ml})$ of $\mathbf{1}(6.8 \mathrm{mg})$ and stirred at room temperature for $24 \mathrm{~h}$. The solution was evaporated under vacuum, residue was purified by silica gel column chromatography using ethyl acetate/petroleum ether (1:9) mixture as eluent. A light yellow solid 2 was obtained, $4.7 \mathrm{mg}$, yield $70 \%$. Compound 2 was crystallized from $\mathrm{MeOH}$ for X-ray analysis. 


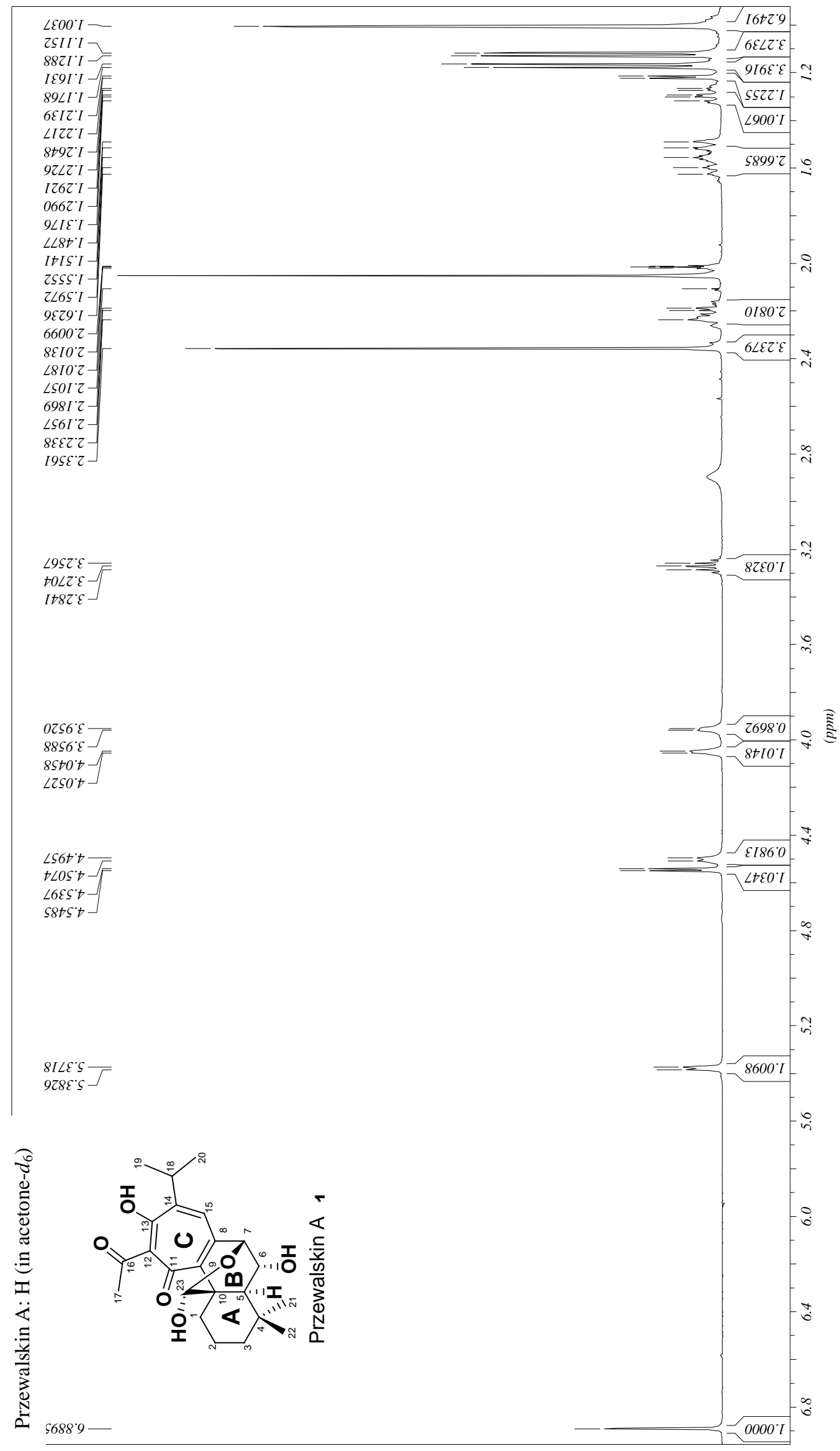




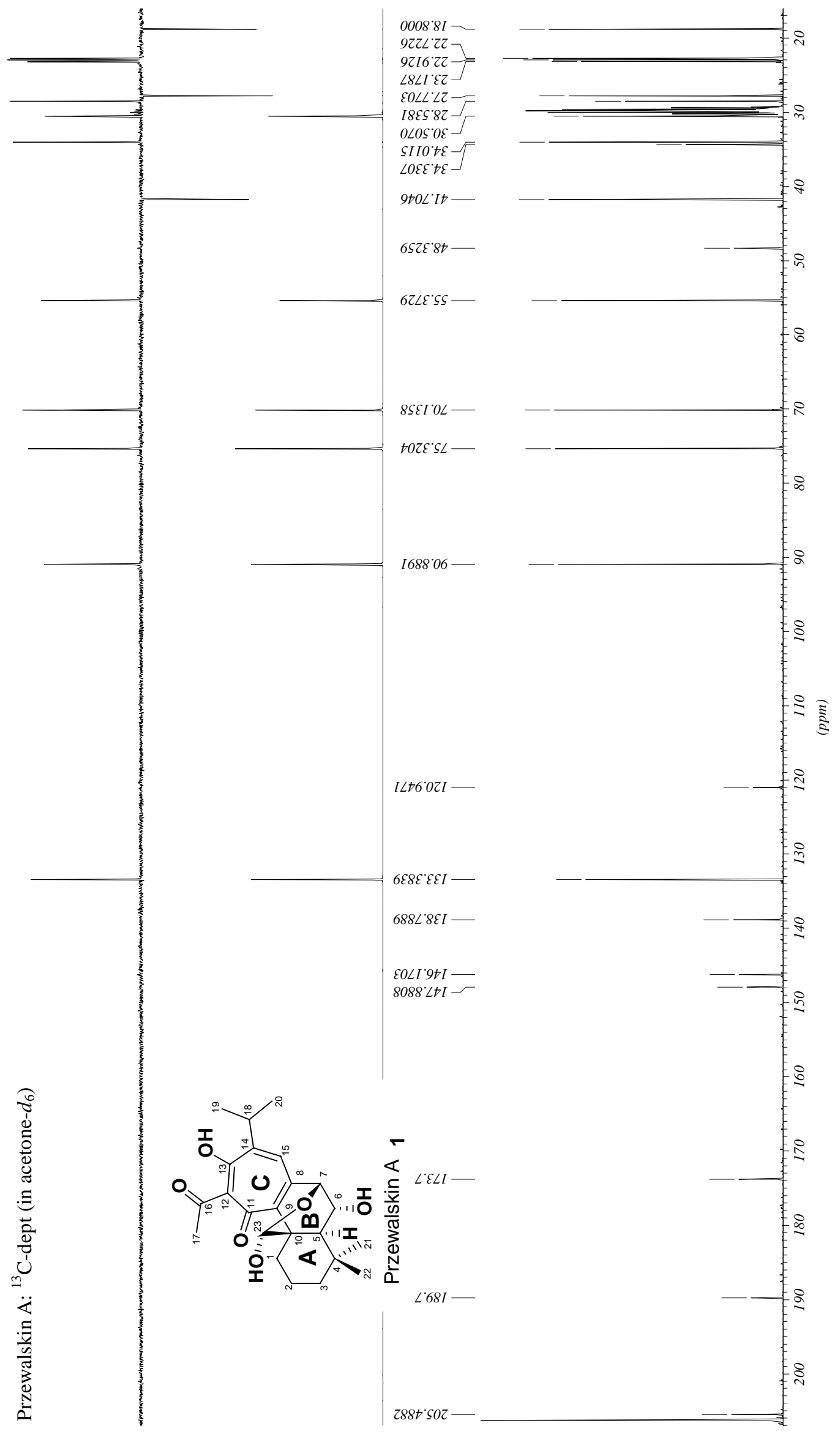




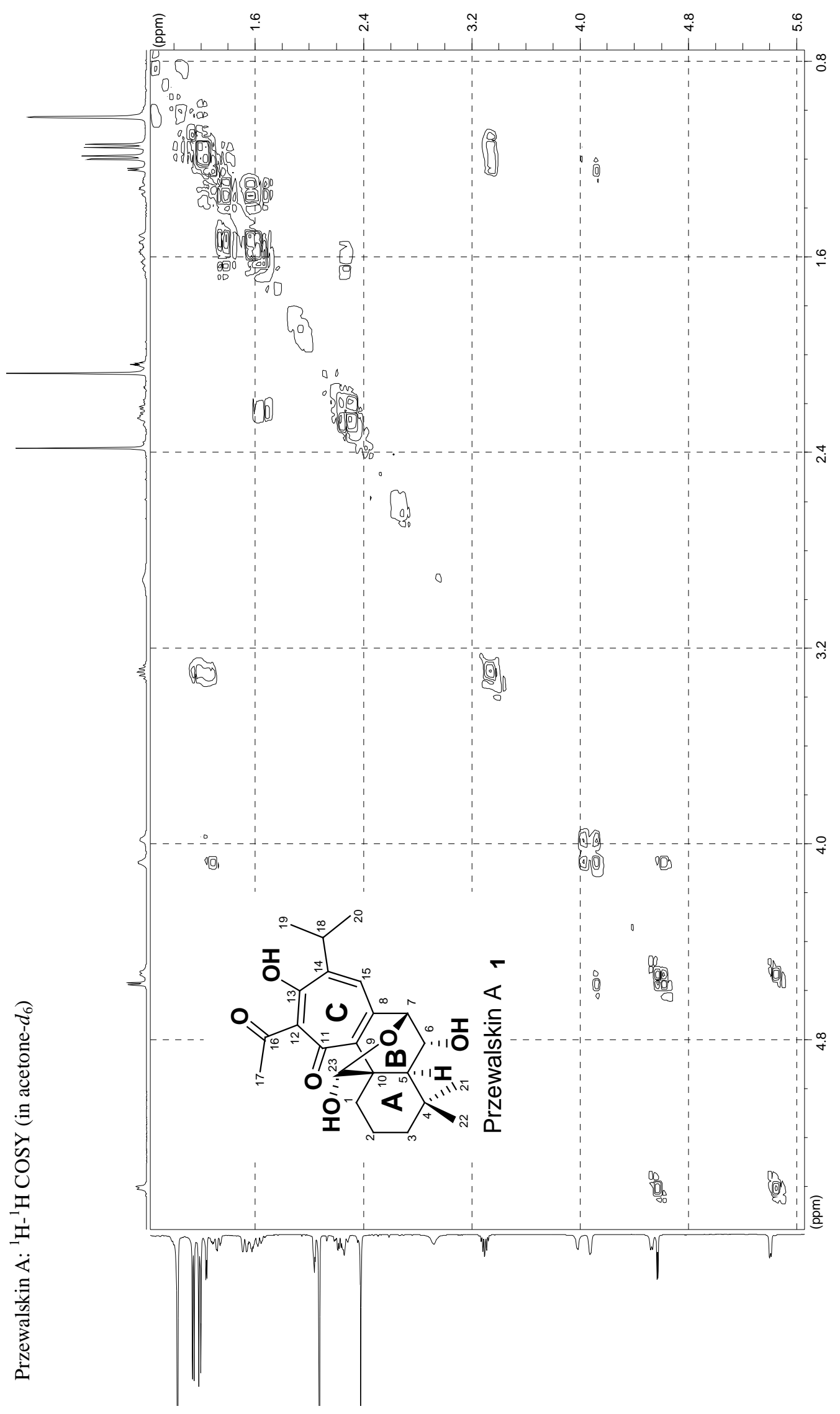




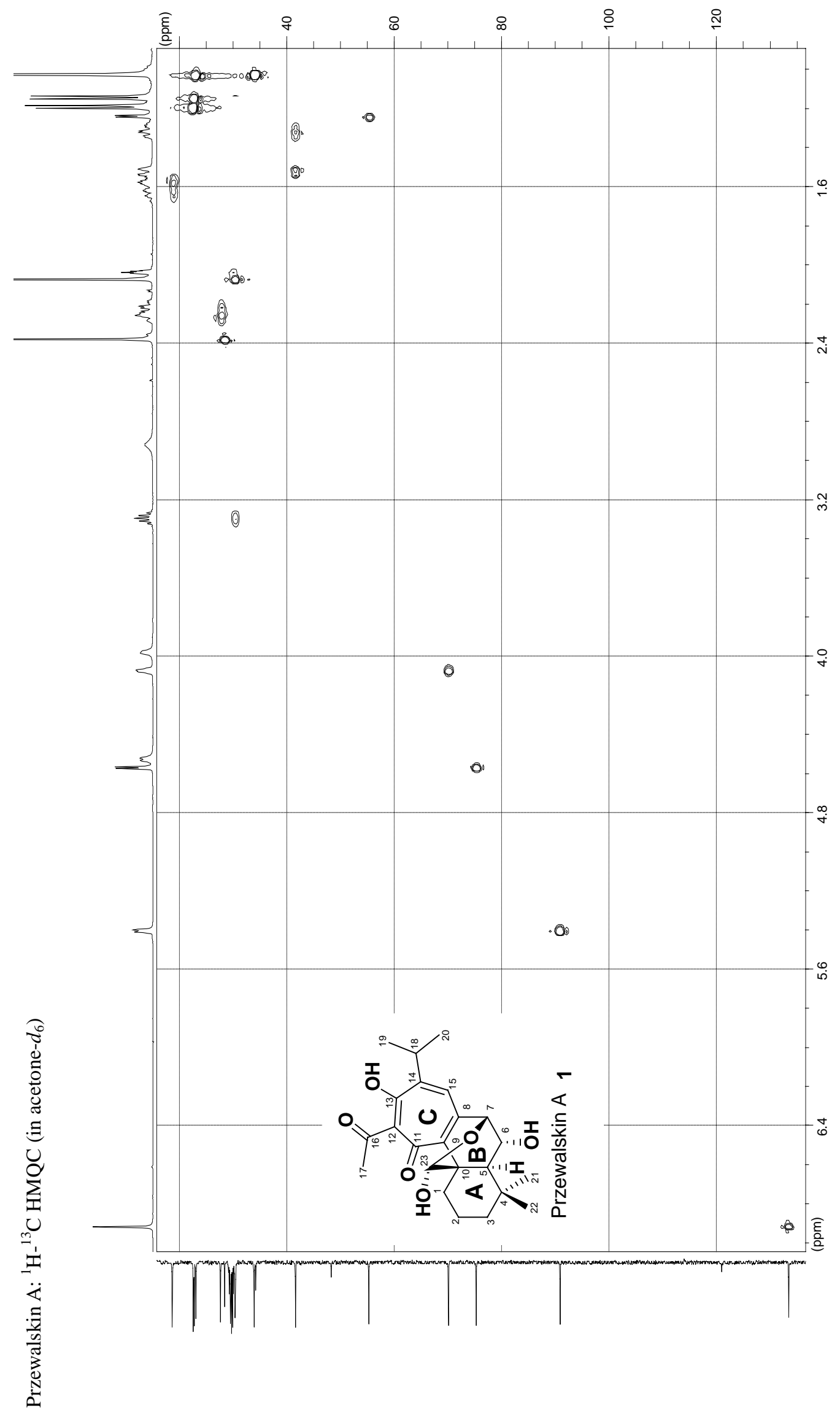




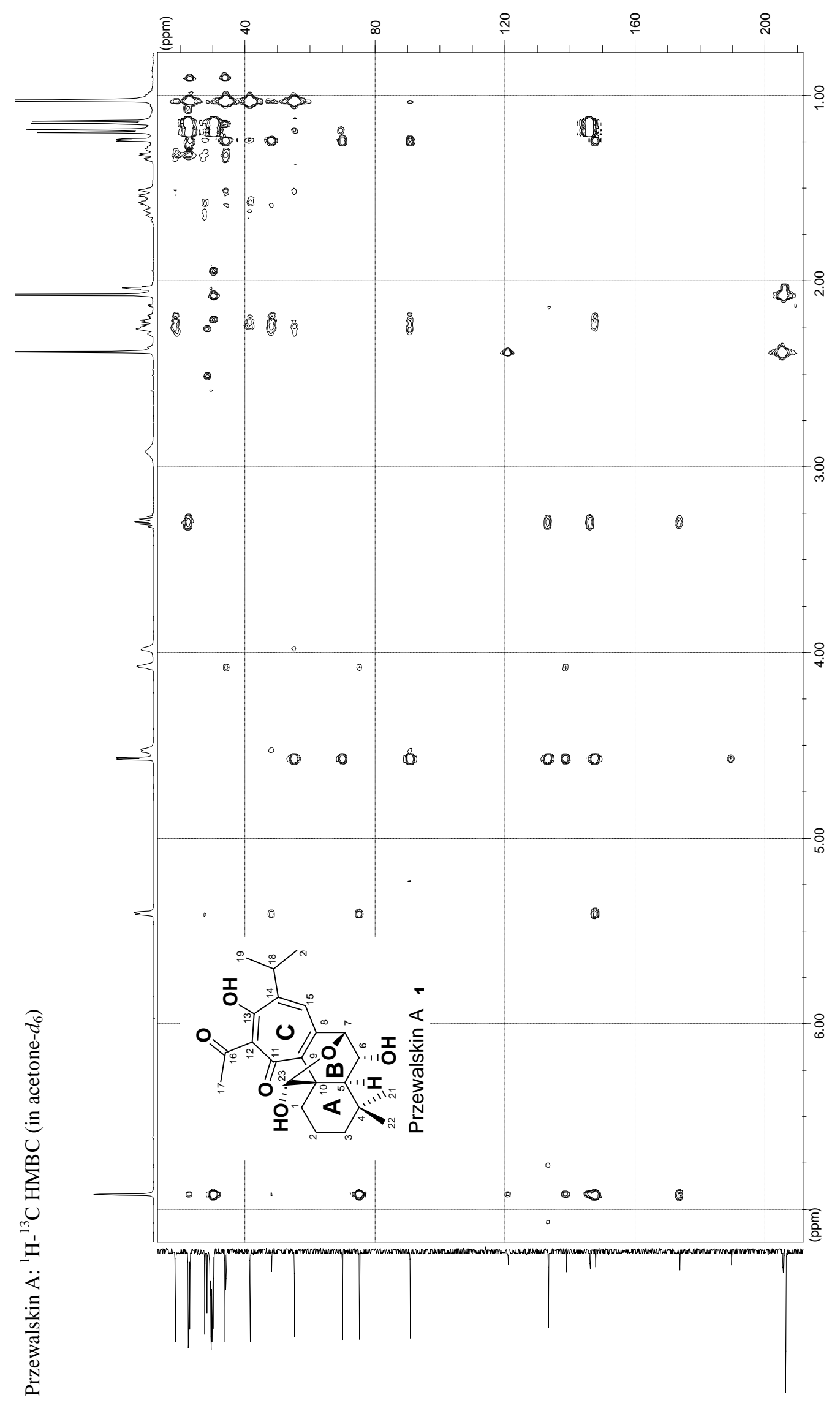




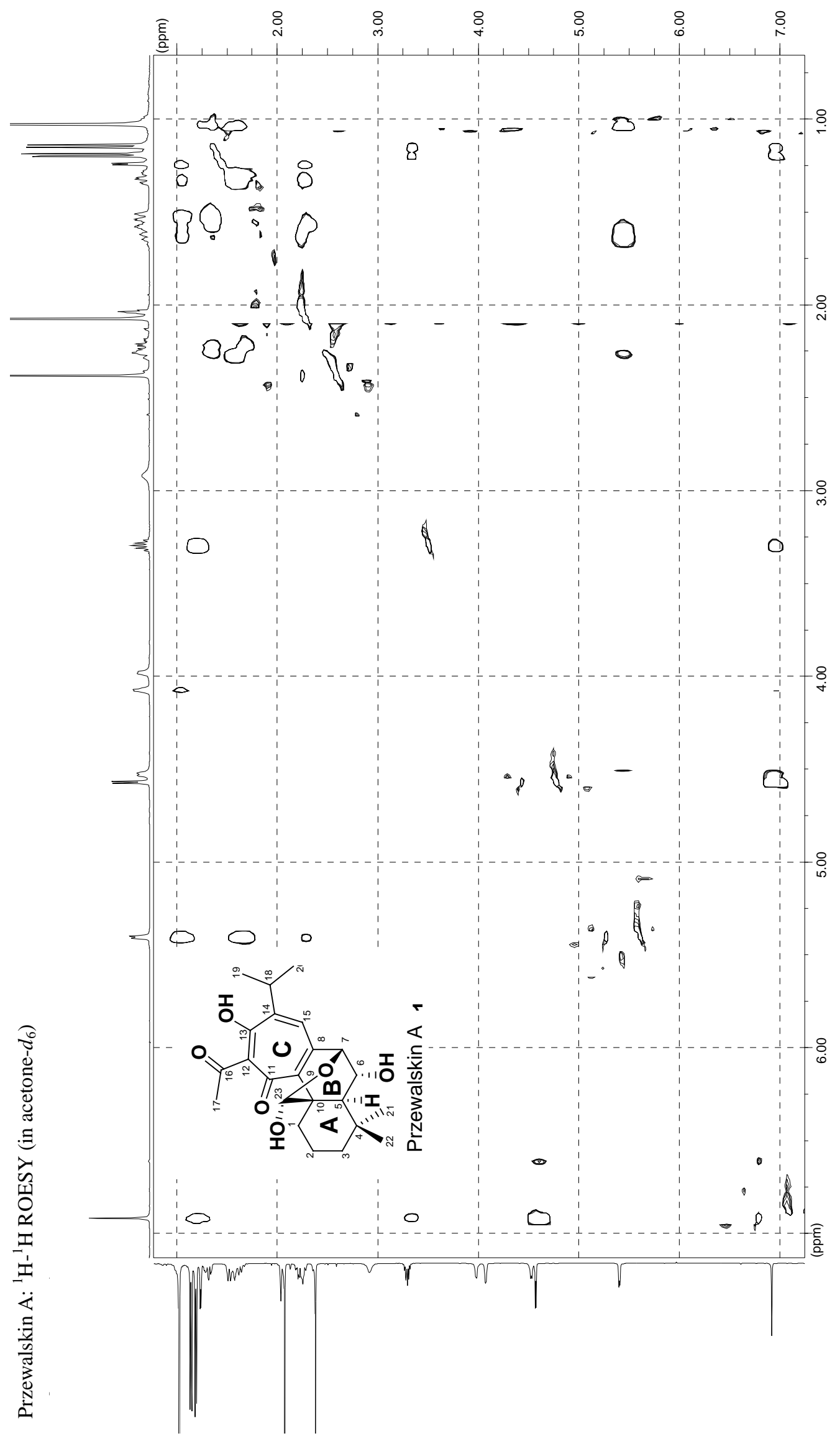




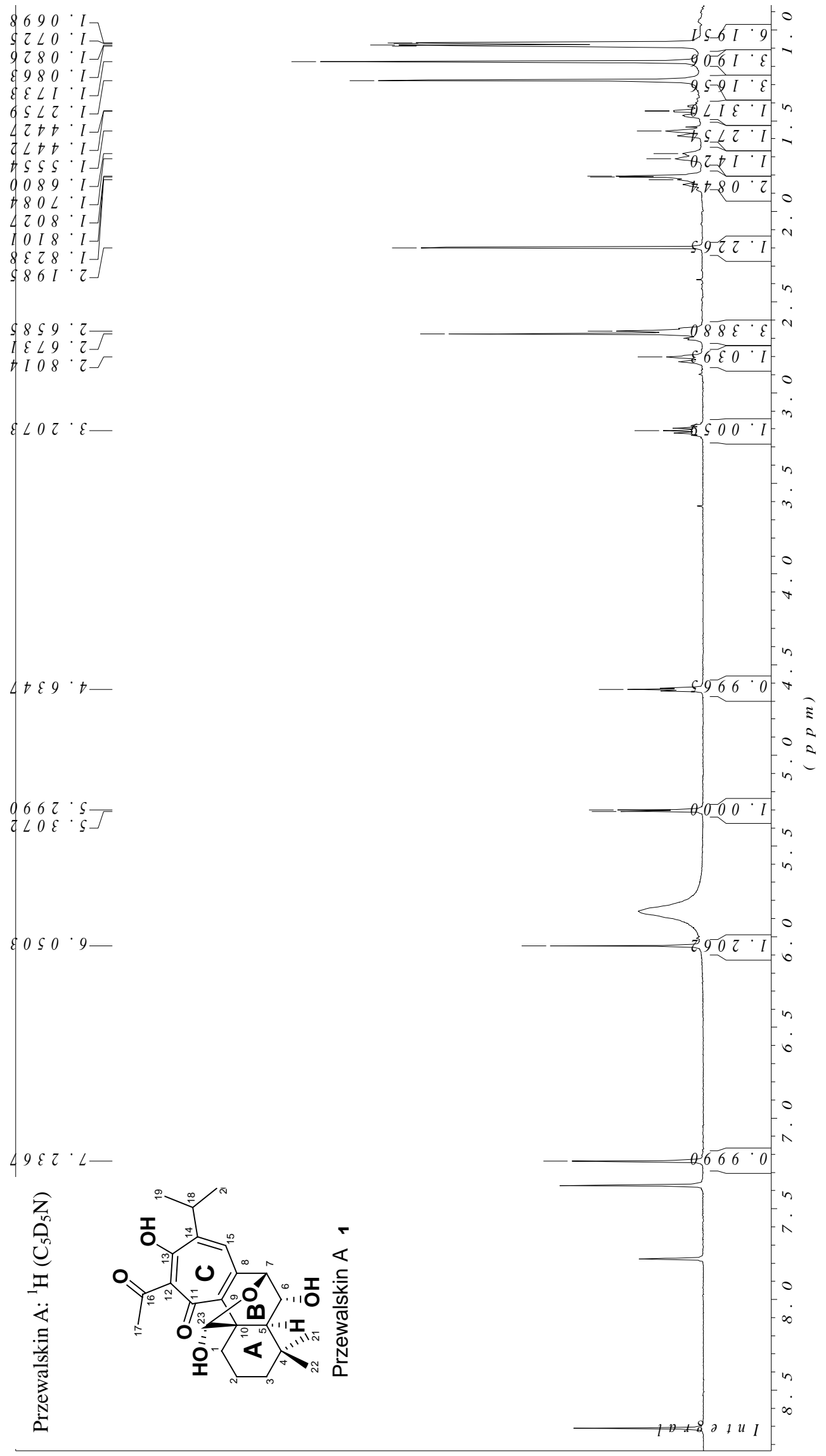




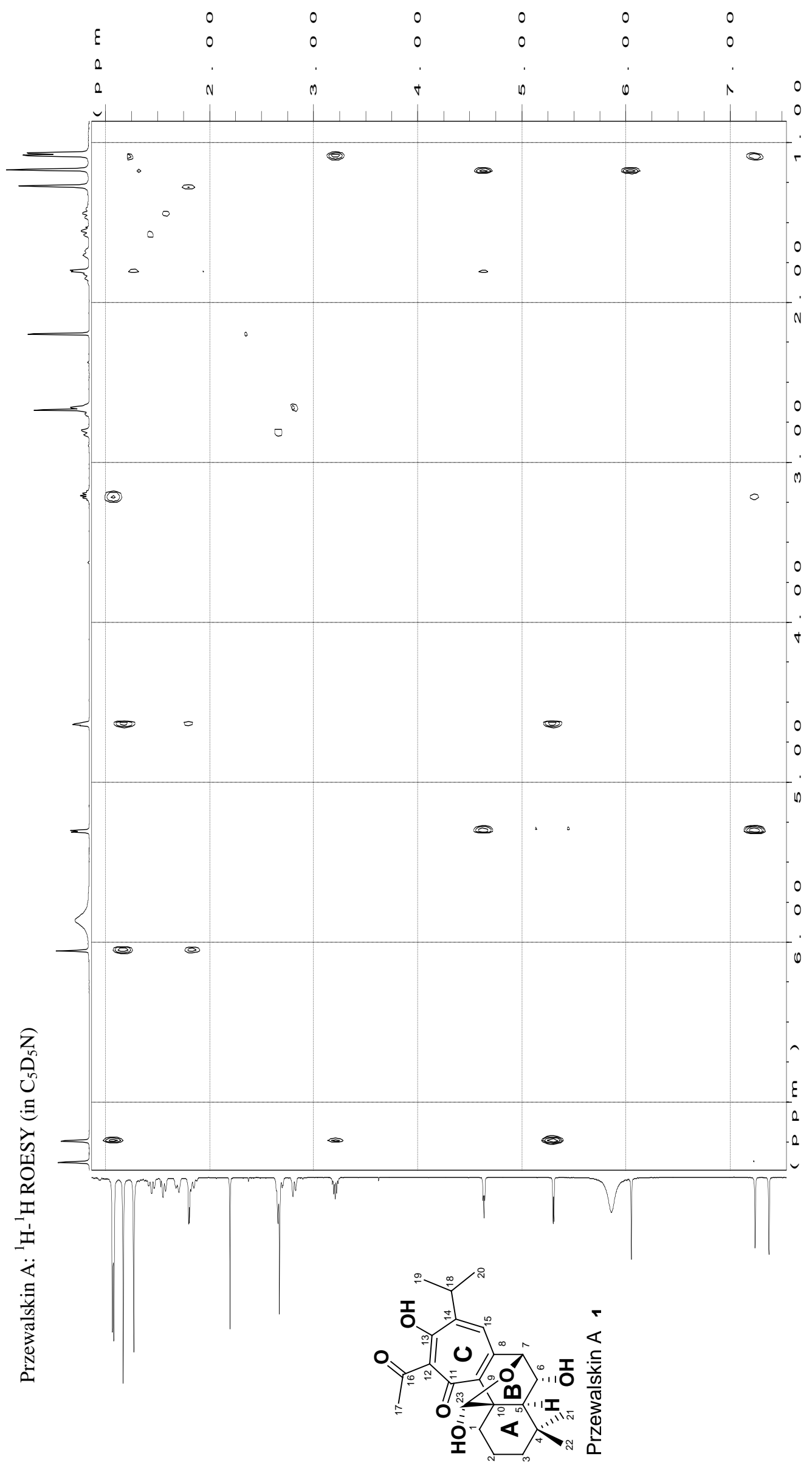




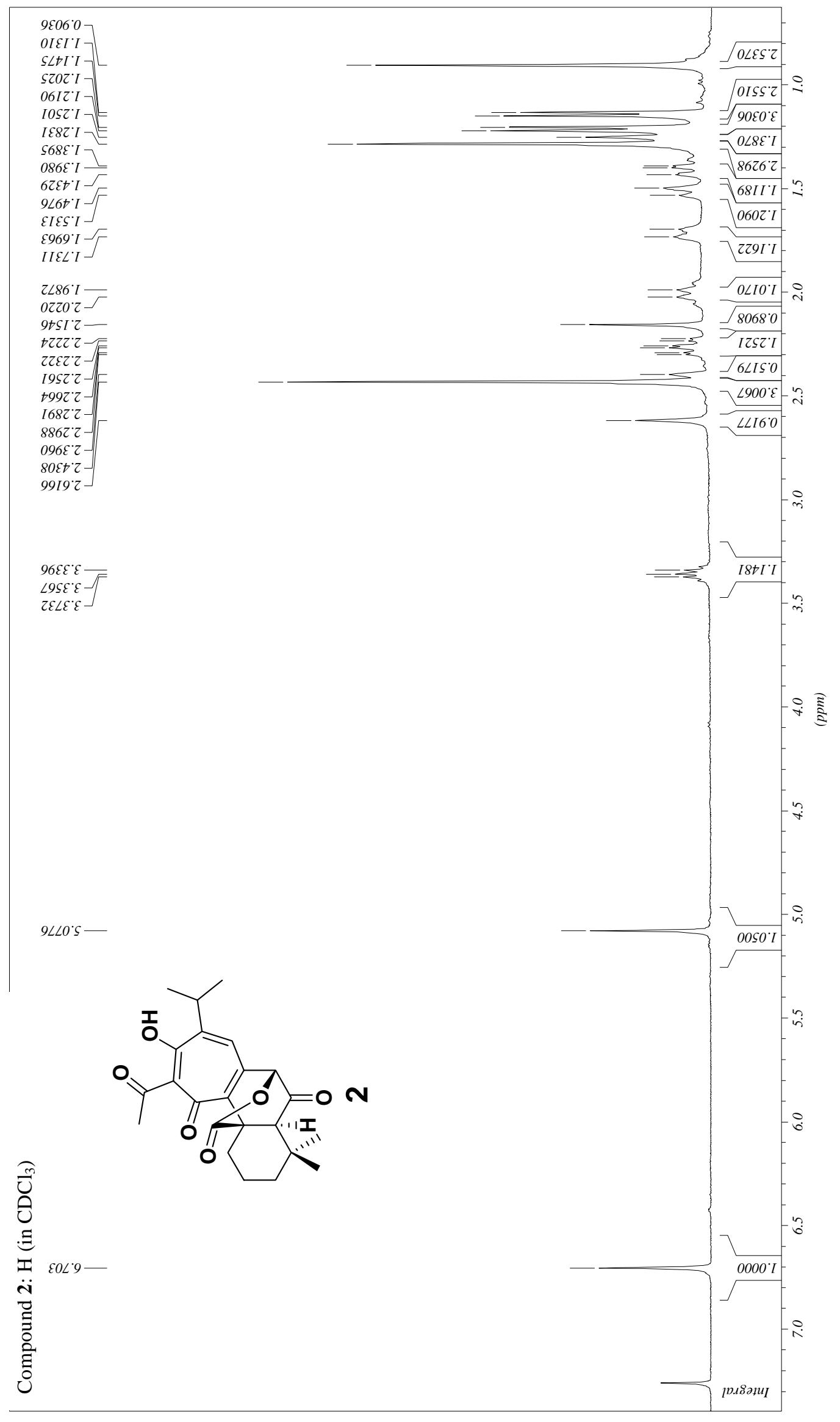




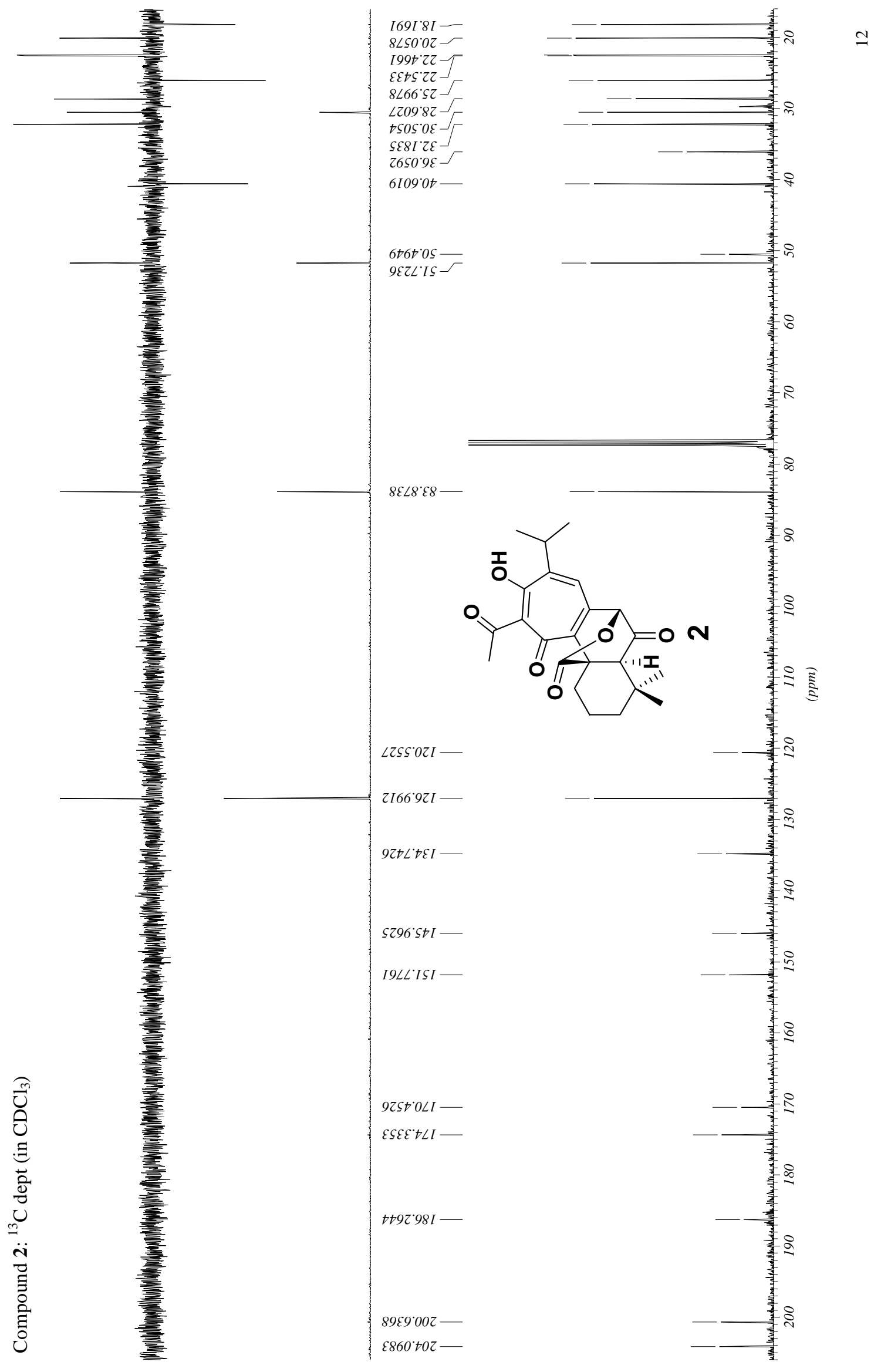




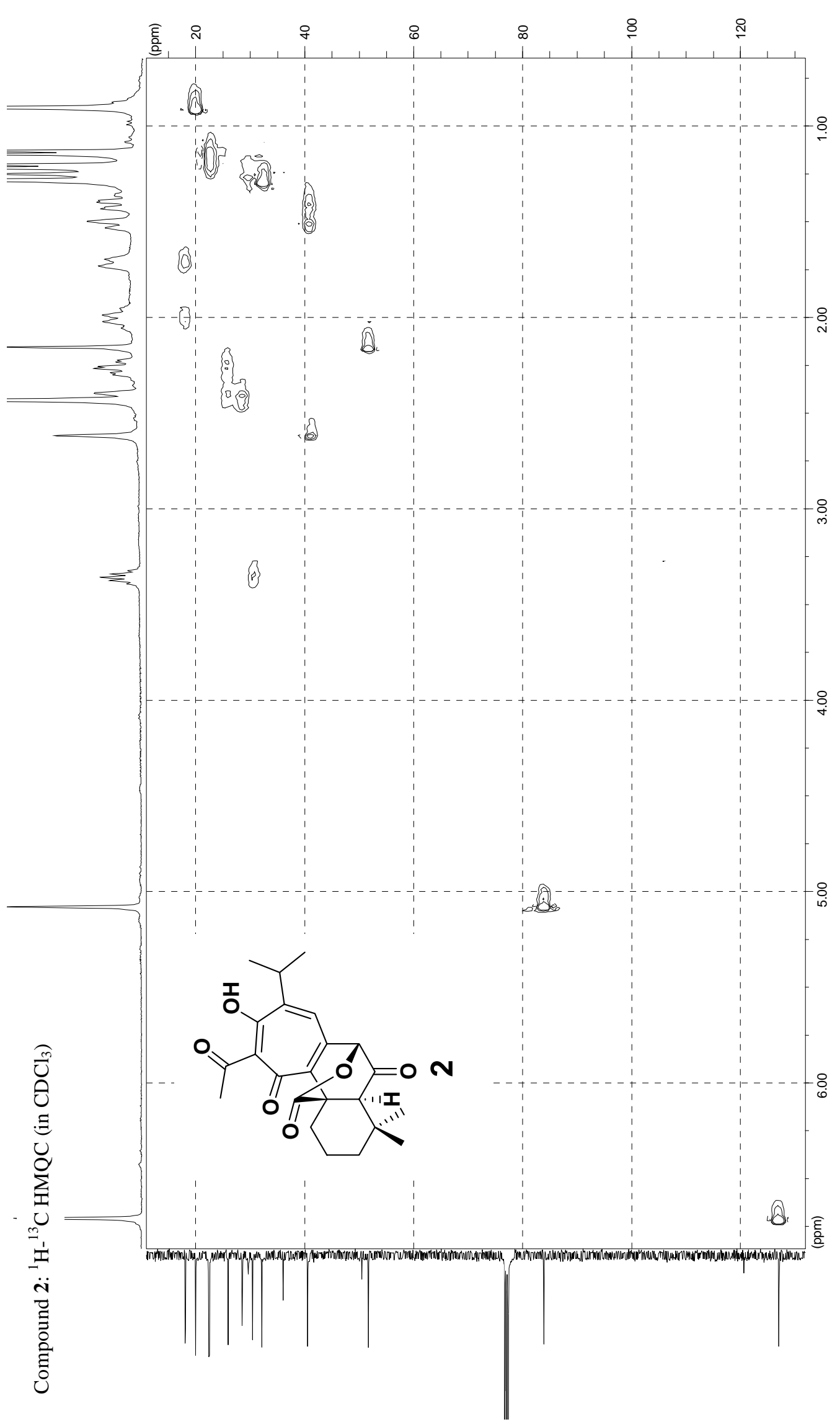




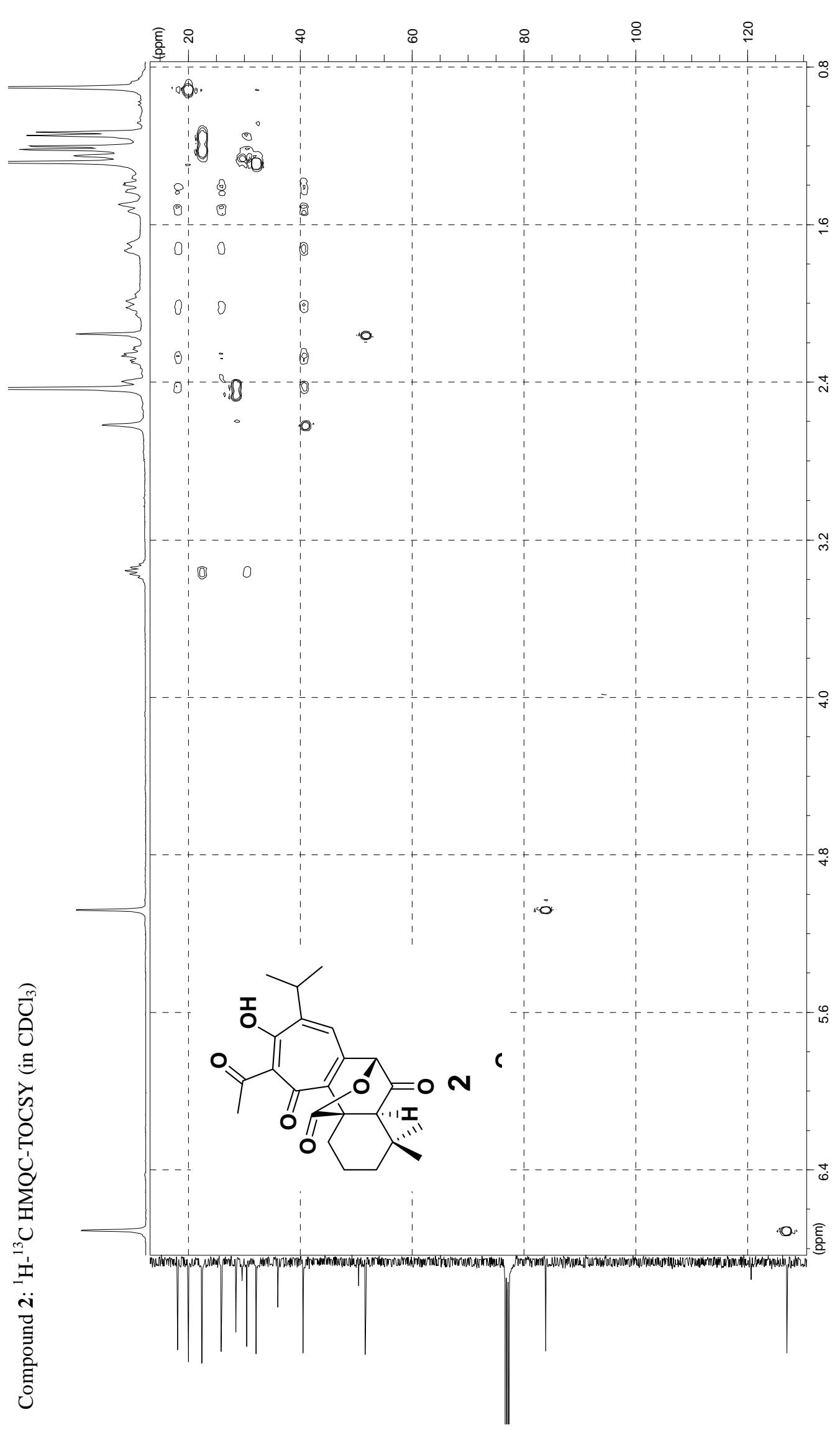




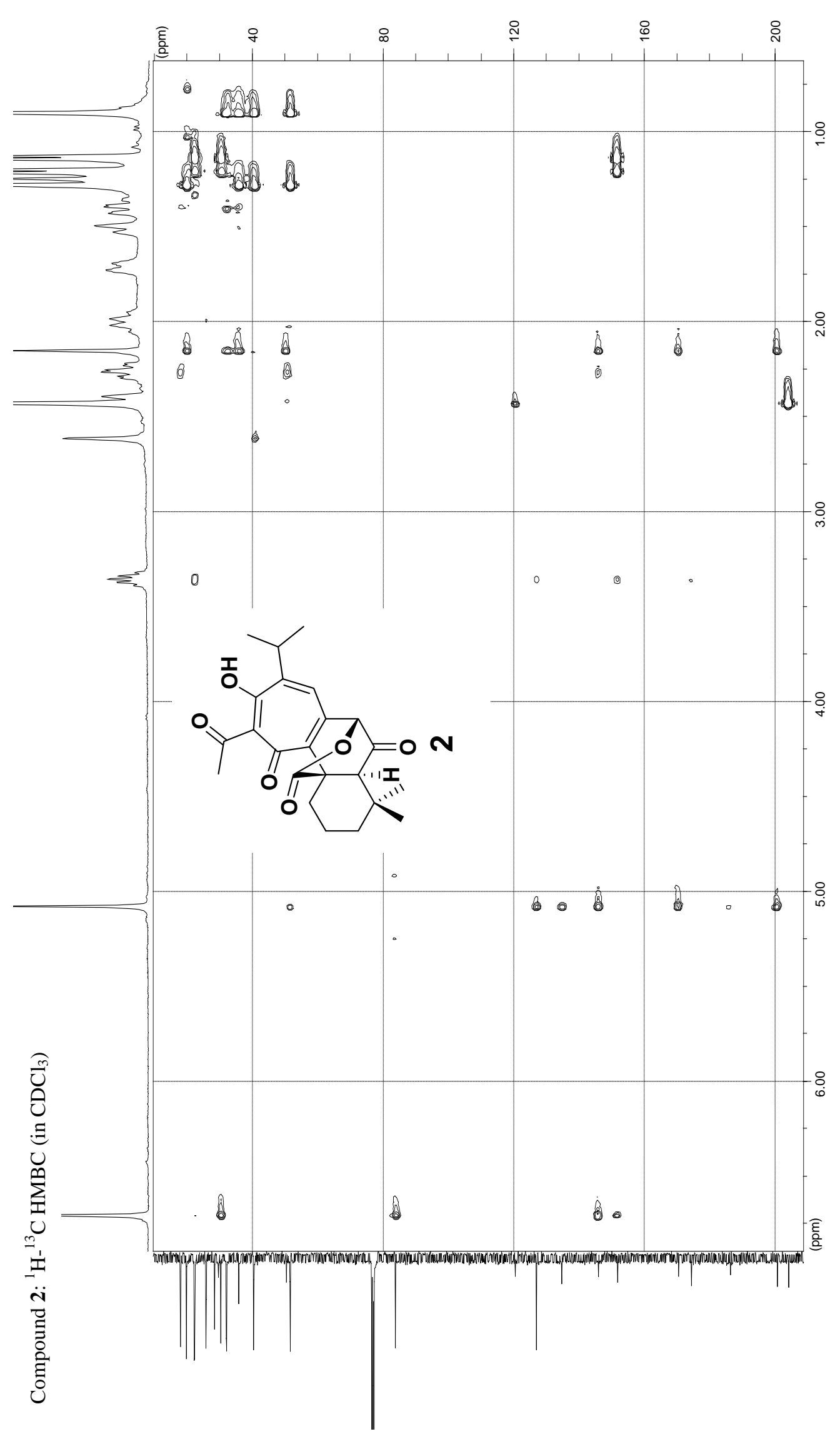




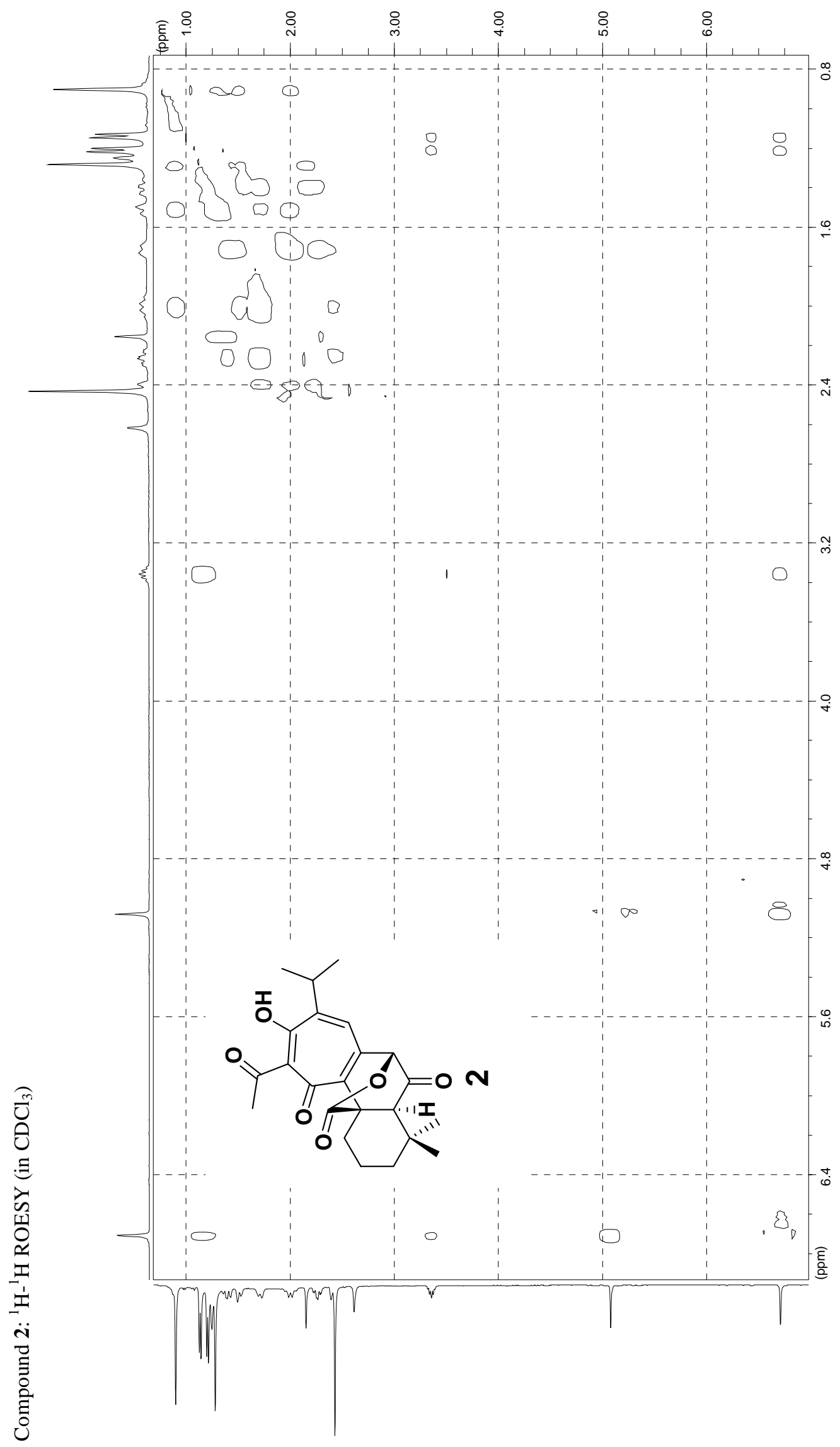




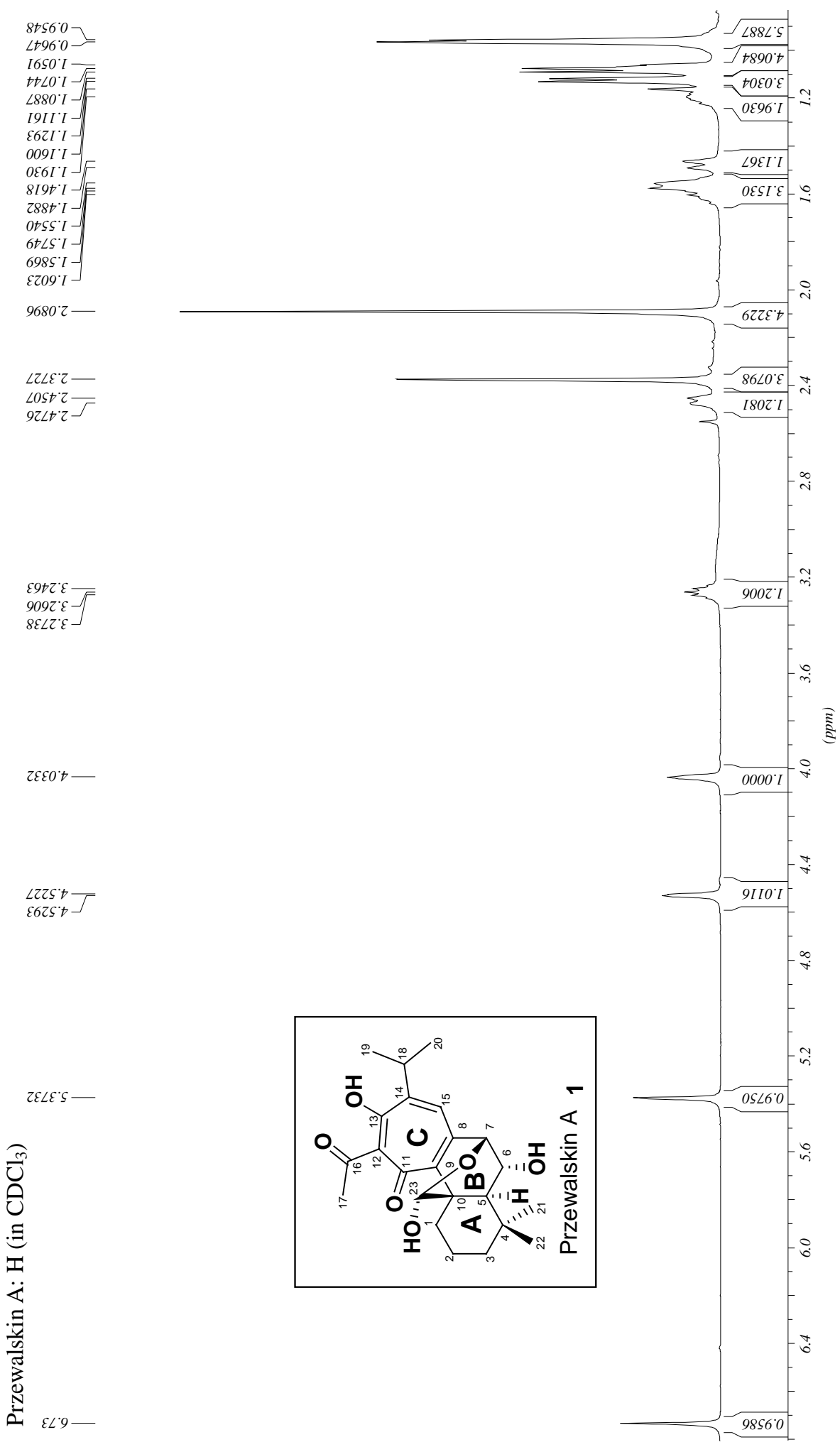




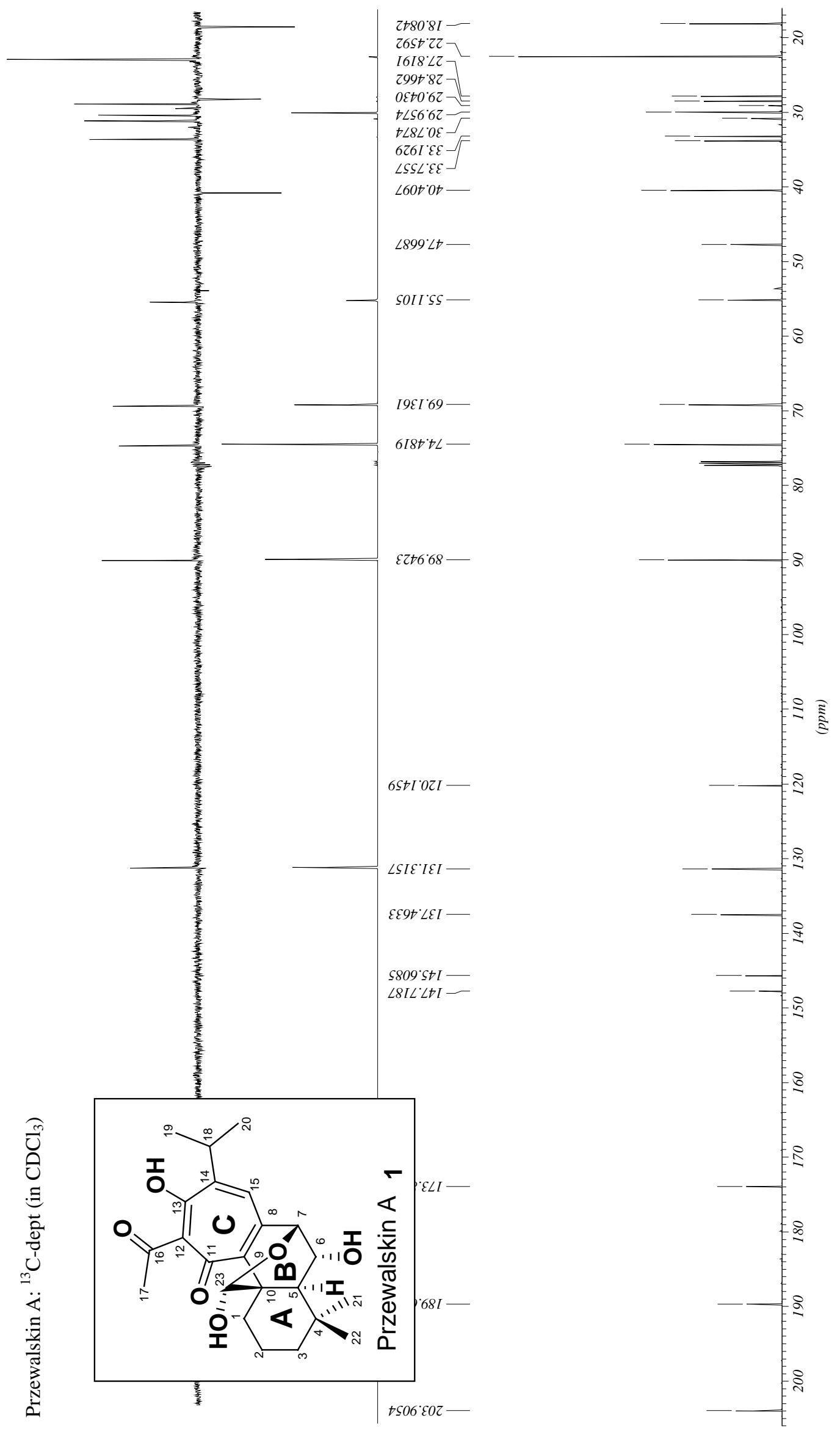




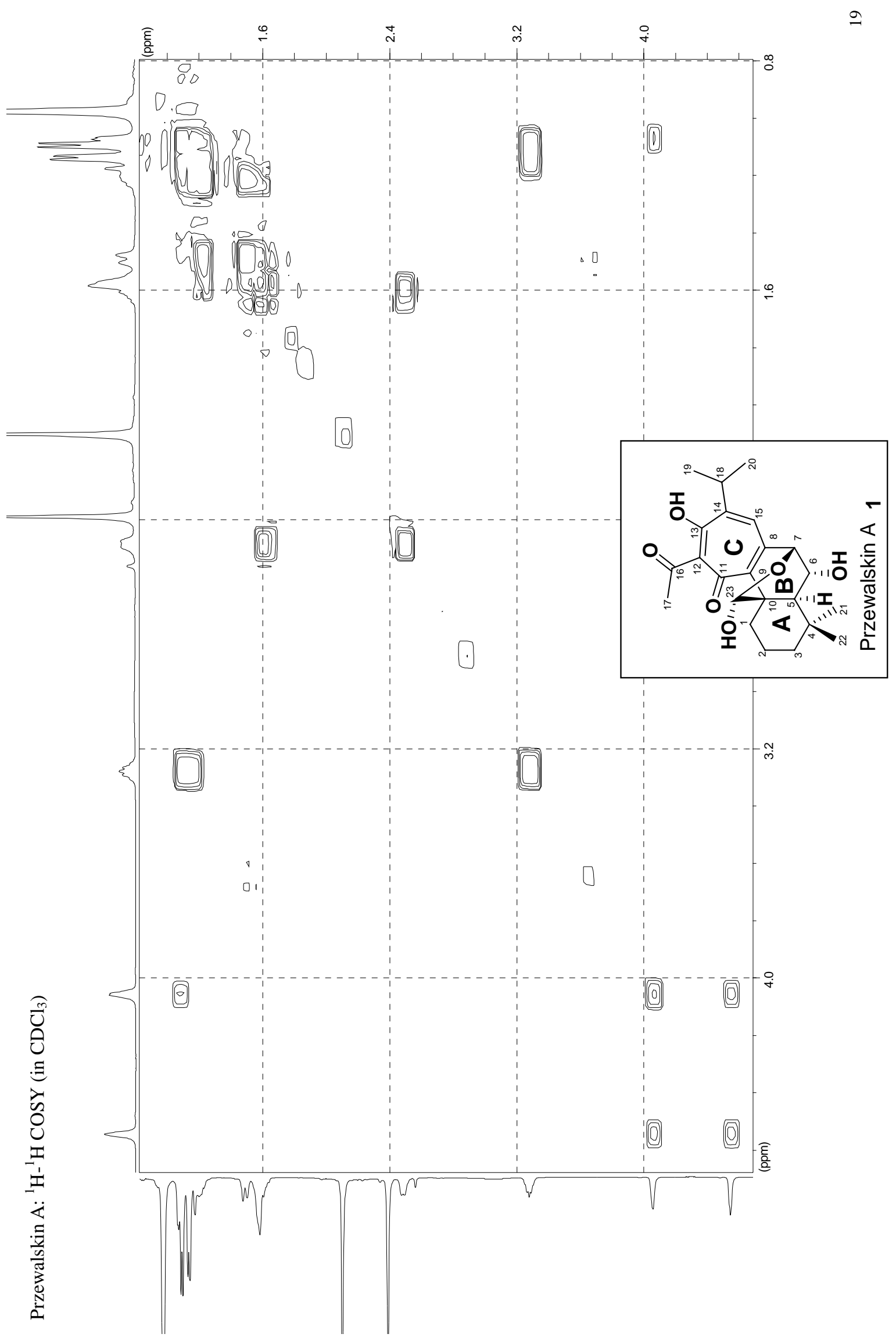




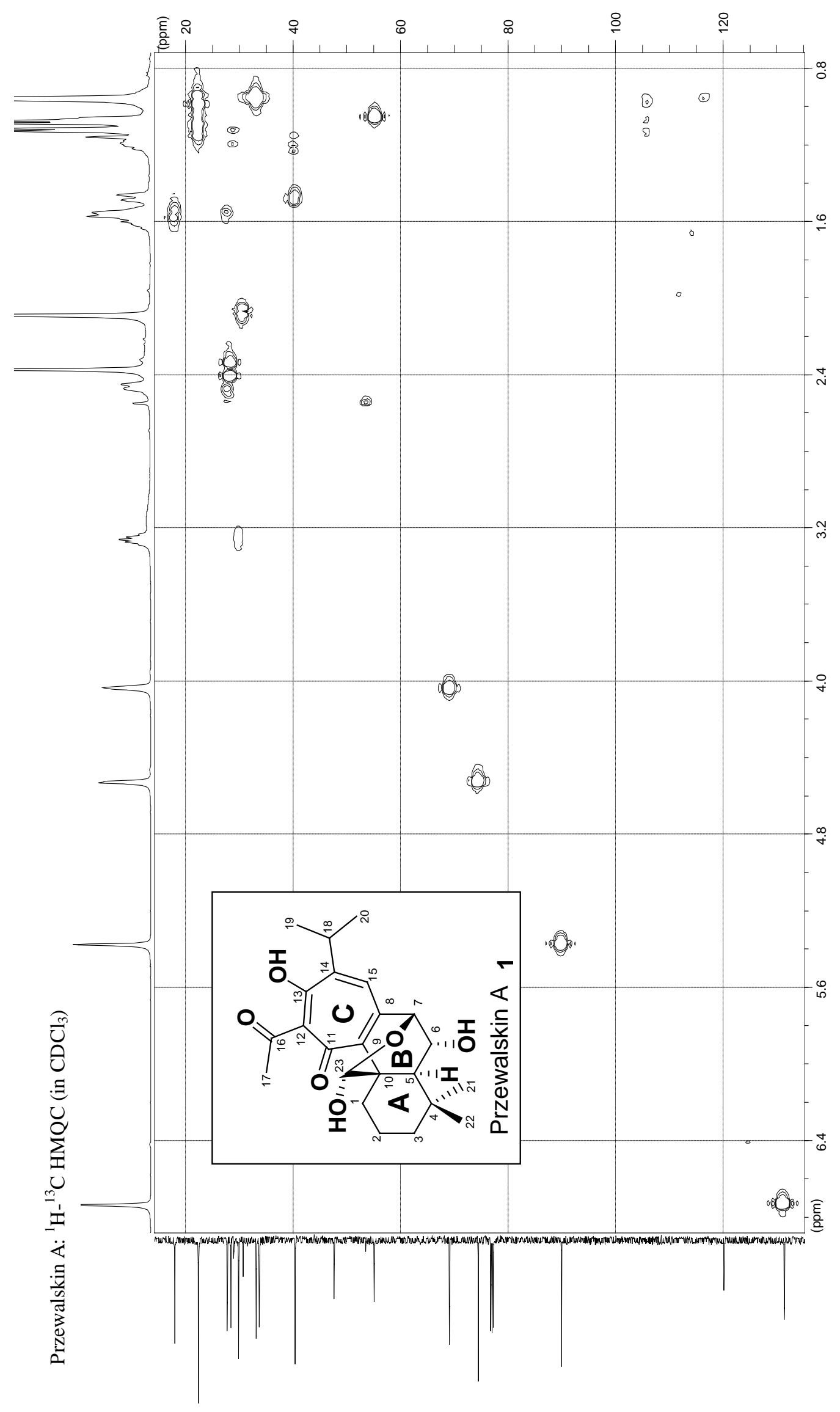




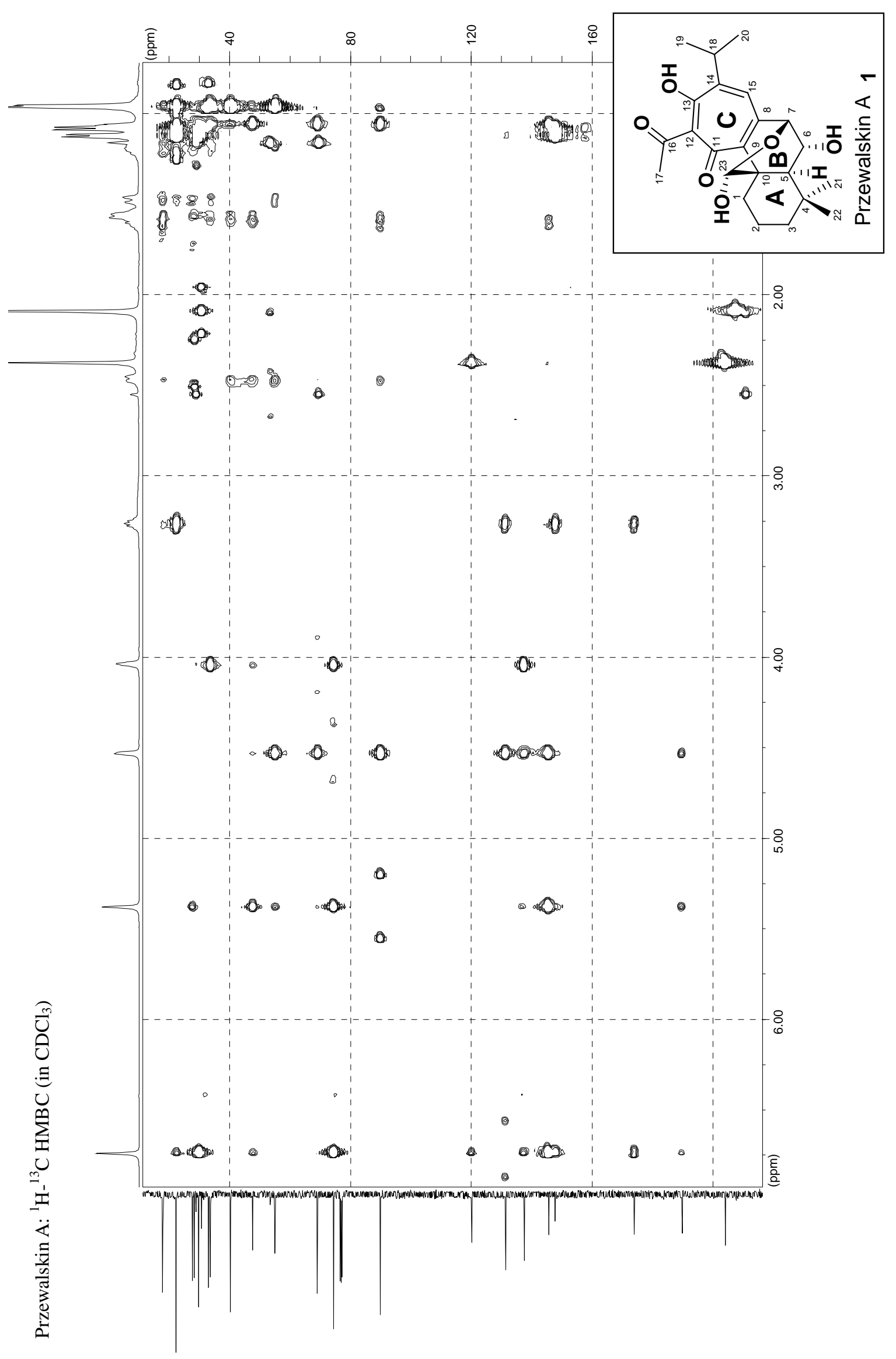

